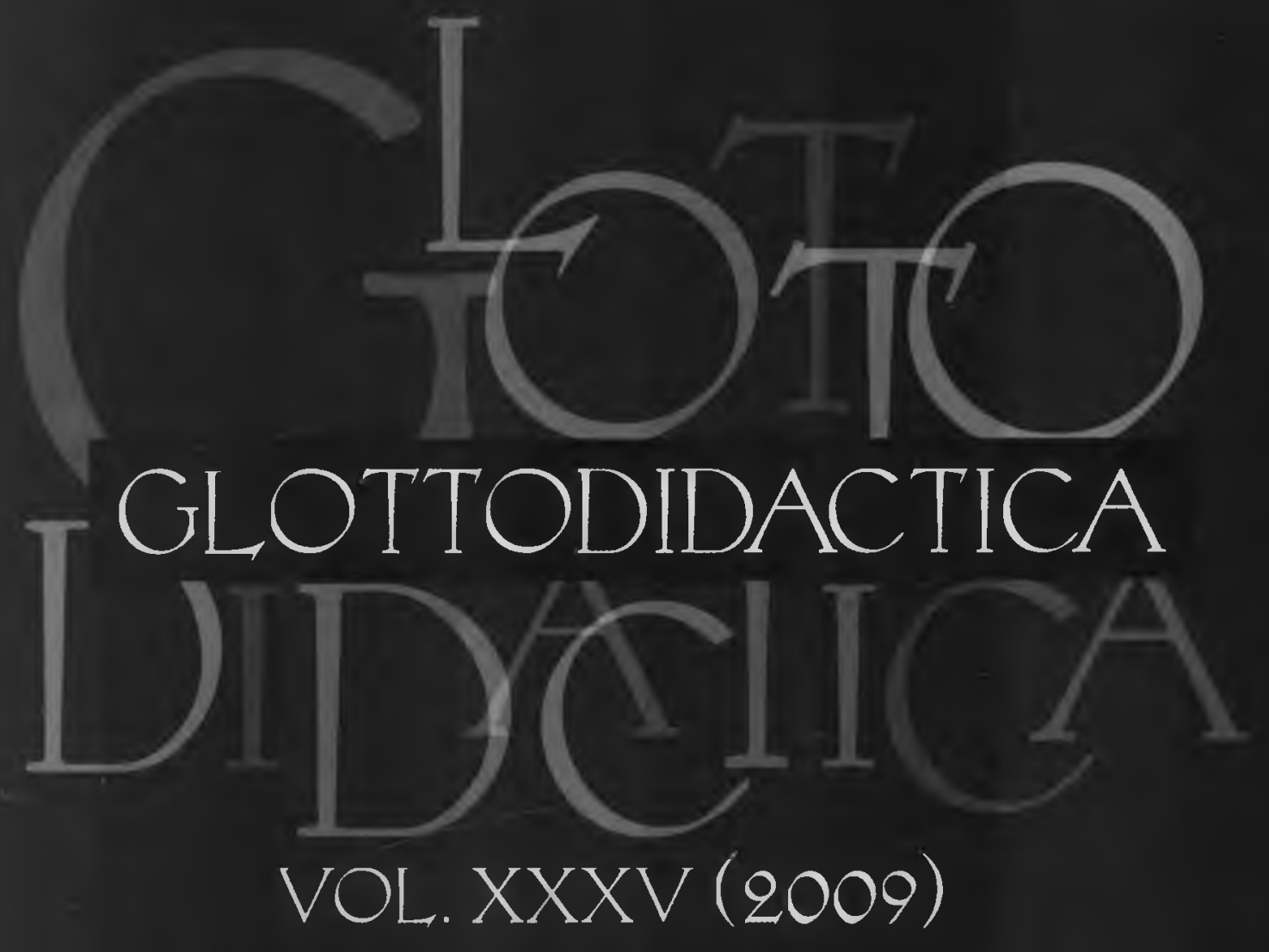

WYDAWNICTWO NAUKOWE UAM 



$$
\operatorname{cod} .42582 \wedge \frac{11}{1309}
$$

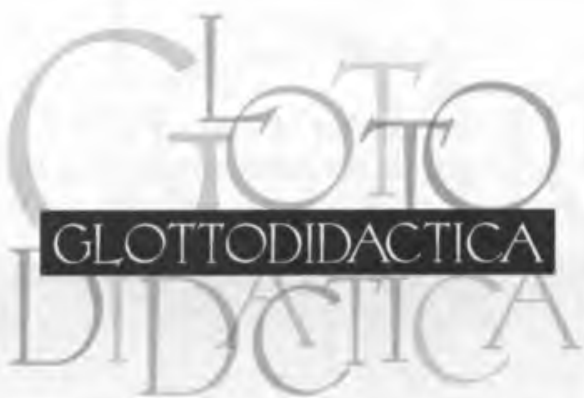




\section{GLOTTODIDACTICA \\ An International Journal of Applied Linguistics \\ Founding Editor: Prof. Dr. Ludwik Zabrocki}

Ausgabe / Issue 35

Herausgeber / Publisher

Institut für Angewandte Linguistik der Adam Mickiewicz Universität Poznań

V.i.S.d.P. Responsible person in accordance with Polish press law

Prof. Dr. Waldemar Pfeiffer (Honorary Editorship)

Redaktion (Leitung) / Editorial Head

Prof. Dr. Barbara Skowronek

Redaktionelle Mitarbeit / Editorial support

Dr. Luiza Ciepielewska-Kaczmarek, Dr. Monika Kowalonek-Janczarek

Redaktionsbeirat Editorial / Advisory Board

Prof. Dr. Jerzy Bańczerowski, Prof. Dr. Anna Cieślicka, Prof. Dr. Józef Darski,

Prof. Dr. Jacek Fisiak, Prof. Dr. Antoni Markunas, Prof. Dr. Kazimiera Myczko,

Prof. Dr. Stanisław Puppel, Prof. Dr. Christoph Schatte, Prof. Dr. Teresa Tomaszkiewicz, Prof. Dr. Weronika Wilczyńska, Prof. Dr. Stephan Wolting, Prof. Dr. Władysław Woźniewicz

Wissenschaftlicher Beirat / Academic Advisory Board

Prof. Dr. Rupprecht S. Baur (Duisburg/Essen), Prof. Dr. Dmitrij Dobrovolskij (Moskau/Wien), Prof. Dr. Franciszek Grucza (Warszawa), Prof. Dr. Wolfgang Herrlitz (Utrecht),

Prof. Dr. Hanna Komorowska (Warszawa), Prof. Dr. Bogdan Kovtyk (Halle),

Prof. Dr. Frank Königs (Marburg/L.), Prof. Dr. Roman Lewicki (Wroclaw),

Prof. Dr. Clare Mar-Molinero (Southhempton), Prof. Dr. Paweł Mecner (Szczecin),

Prof. Dr. Bernd Müller-Jacquier (Bayreuth), Prof. Dr. David S. Singleton (Dublin), Prof. Dr. Marian Szczodrowski (Gdańsk), Prof. Dr. Iwar Werlen (Bern), Prof. Dr. Jerzy Żmudzki (Lublin) 
UNIWERSYTET IM. ADAMA MICKIEWICZA W POZNANIU

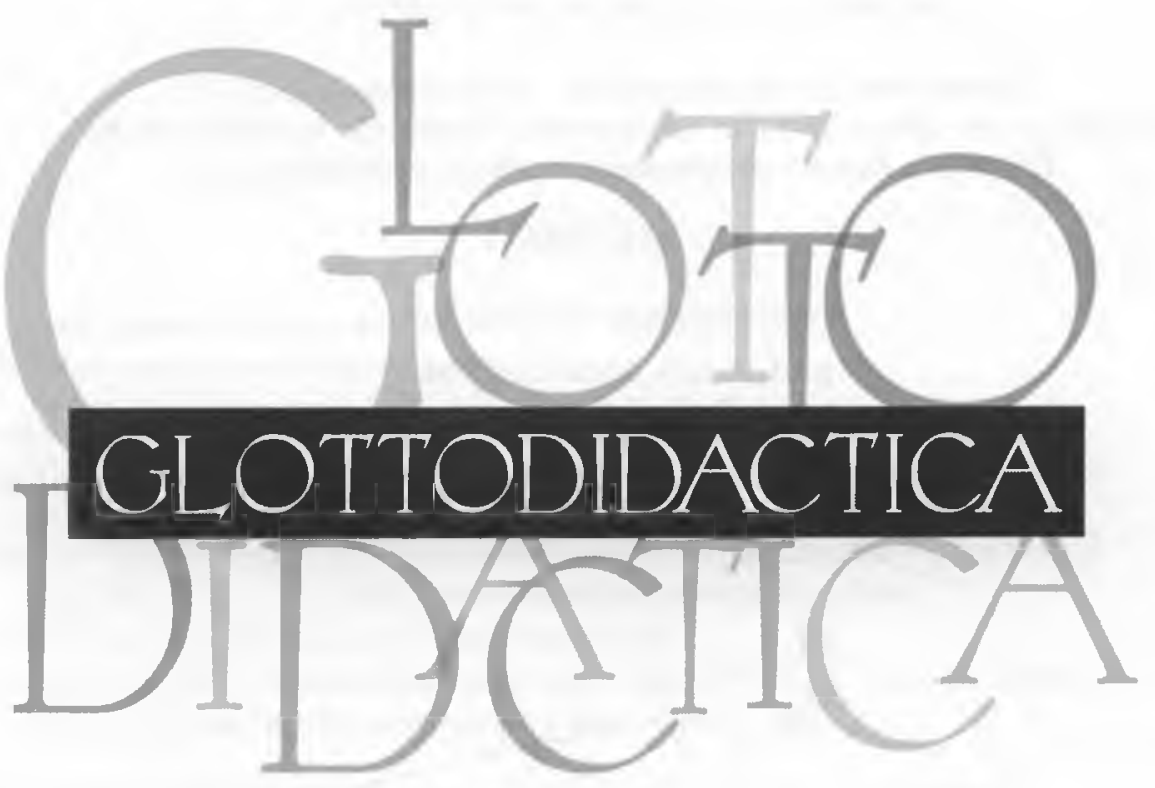

VOLUME XXXV

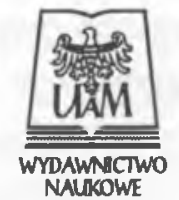

POZNAŃ 2009 


$$
\begin{gathered}
\text { Adres redakcji } \\
\text { Instytut Lingwistyki Stosowanej UAM } \\
\text { ul. } 28 \text { Czerwca } 1956 \text { r. nr } 198 \\
\text { 61-485 Poznań, Poland } \\
\text { tel. }+48618292925 \text {, tel./ fax }+48618292926
\end{gathered}
$$

Editor: Prof. Barbara Skowronek barbaras@amu.edu.pl Assistants to the Editor: Dr Luiza Ciepielewska-Kaczmarek luizac@poczta.fm

Dr Monika Kowalonek-Janczarek monika.kowalonek@wp.pl

$$
\begin{gathered}
\text { Weryfikacja językowa tekstów } \\
\text { prof. dr hab. Anna Cieślicka } \\
\text { dr Britta Stöckmann }
\end{gathered}
$$

Publikacja dofinansowana przez

Instytut Lingwistyki Stosowanej UAM

\section{POZNANI}

(C) Wydawnictwo Naukowe UAM, Poznań 2009

Wydano na podstawie maszynopisu gwarantowan go

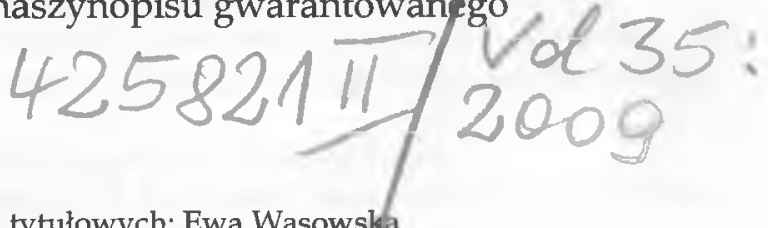

Projekt okladki i stron tytułowych: Ewa Wąsowska

Redaktor prowadzący: Anna Rąbalska

Formatowanie: Eugeniusz Strykowski

\section{ISBN 978-83-232-2083-1 ISSN 0072-4769}

WYDAWNICTWO NAUKOWE UNIWERSYTETU IM. ADAMA MICKIEWICZA

61-734 Poznań, ul. F. Nowowiejskiego 55, tel. 06182939 85, fax 0618293980 e-mail: press@amu.edu.pl www.press.amu.edu.pl

Ark. wyd. 17,00 . Ark. druk. 15,50

ZAKŁAD GRAFICZNY UAM, POZNAŃ, UL. H. WIENIAWSKIEGO 1

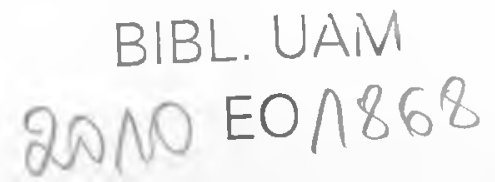




\section{CONTENTS}

\section{ARTICLES}

Basic and Referential Disciplines

Grundlagen- und Referenzwissenschaften

MARIAN SZCZODROWSKI, Wesen, Arten und Wirkungen der fremdsprachlichen Steuerung ...

GRAŻYNA LEWICKA, Zu einigen Problemen des Verstehens in der konstruktivistisch orientier-

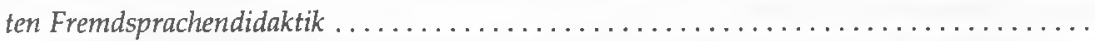

REINHOLD UTRI, Bilinguale Erziehung - neue Herausforderungen und Lösungen aus linguisti-

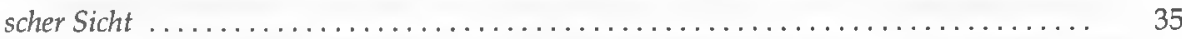

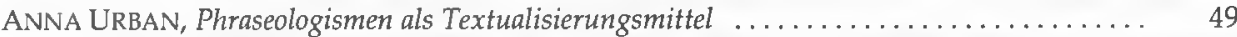

NATASCHA JOURDY, НарратиВная структура художественного текста: свободнокосвенный дискурс как категория лингвистики и нарратологии ................

\section{Methodology in Glottodidactics}

Fremdspracheninethodik

ZOFIA CHLOPEK, The relationship between language learning experience, motivation and some

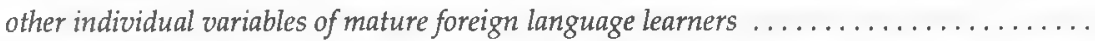

MALGORZATA CZARNECKA, Die Entwicklung interner grammatischer Regeln im gesteuerten

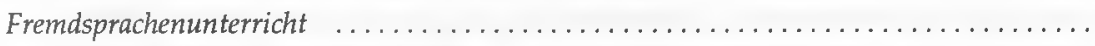

BRIGITA KOSEVSKI PULJIĆ, Der Enwerb der Schreibkompetenz im Deutsch als Fremdsprache -

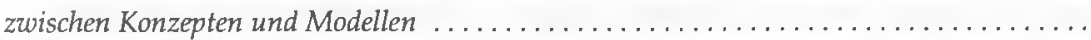

AGNIESZKA MAC, Einige Überlegungen zur Wortbildungslehre im fremdsprachlichen

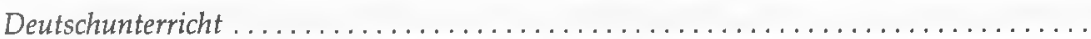

SYlWIA ADAMCZAK-KRYSZTOFOWICZ, ANTJE STORK, Podcasts im glottodidaktischen Medienverbund. Versuch einer vergleichenden Analyse ......................

MARIUSZ WALORCZYK, Optimierung der ZD-Prüfungsvorbereitung in Bezug auf die Aufgabenformen

\section{Language Policy and Intercultural Studies}

Sprachenpolitik und Interkulturelle Studien

MONIKa KOWALONEK-JANCZAREK, Die Nachbarsprache Polnisch aus einer sprachpolitischen und soziolinguistischen Perspektive im Kontext der Mehrsprachigkeit in Europa .........

NINO LOLADZE, Interkulturelles Lernen als Schlüsselqualifikation im Zeitalter der Globalisierung im Kontext des Deutschunterrichts in Georgien 


\section{RESEARCH REPORTS}

DANUTA WIŚNIEWSKA, EFL Teachers' Perception and Practice of Action Research .......... 163

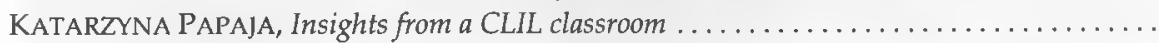

MAGDAlENA KOPER, MAGDALENA DUdZIŃSKA, Europäisierung der Sprachenpolitik im Bereich des Kulturtourismus nach dem EU-Beitritt Polens am Beispiel der Stadt Gdansk ....

\section{BOOK REVIEWS}

BARBARA SKOWRONEK, Marian Szczodrowski: Fremdsprachliche Lehr-Lern-Vorgange im kodematischen Blickfeld. Wydawnictwo Uniwersytetu Gdańskiego, Gdańsk - Sopot 2009,225 S. . . . . . . . . . . . . . . . . . . . . . . . . . . . . . .

JOANNA KIC-DRGAS, Sambor Grucza: Lingwistyka języków specjalistycznych. Wydawnictwo Euro-Edukacja, Warszawa 2008, 256 S.

ANNA PIECZYNSKA-SUlIK, Hans-Jörg Schwenk: Präfigierung im Polnischen und ihre Entsprechung im Deutschen. $\mathrm{Zu}$ Theorie von und lexikographischem Umgang mit Aspekt und Aktionsart. Reihe: Języki - Kultury - Teksty - Wiedza. Band 8, Wydawnictwo EuroEdukacja, Warszawa 2007, $301 \mathrm{~S}$.

BARBARA SKOWRONEK, Magdalena Olpińska: Nauczanie dwujęzyczne w świetle badań $i$ koncepcji glottodydaktycznych. Seria: Języki - kultury - teksty - wiedza. Franciszek Grucza, Jerzy Lukszyn (Hg.). Wydawnictwo Euro-Edukacja, Warszawa 2008, 247 S. . . . . . .

REINHOLD UTRI, Marc Bielefeld: We spe@k Deutsch... aber verstehen nur Bahnhof. Unterwegs im Dschungel unserer Sprache. Wilhelm Heyne Verlag, München, 2008, 286 S. .

GABRIELA GORĄCA, Sambor Grucza: Od lingwistyki tekstu do lingwistyki tekstu specjalistycznego. Seria: Języki - kultury - teksty - wiedza. Franciszek Grucza, Jerzy Lukszyn (Hg.). Wydawnictwo Euro-Edukacja, Warszawa 2007, 218 S.

KATARZYNA KONSEK, Jörg Roche: Handbuch Mediendidaktik. Hueber Verlag, Ismaning $2008,176 \mathrm{~S}$.

ANNA URBAN, Christiane Hümmer: Synonyme bei phraseologischen Einheiten. Eine korpusbasierte Untersuchung. Peter Lang, Frankfurt am Main 2009, 357 S. . . . . . . . .

SYLWIA ADAMCZAK-KRYSZTOFOWICZ, Kazimiera Myczko, Barbara Skowronek, Władyslaw Zabrocki (red.): Perspektywy glottodydaktyki $i$ jezykoznawstwa. Tom jubileuszowy $z$ okazji 70. urodzin Profesora Waldemara Pfeiffera. Wydawnictwo Naukowe UAM, Poznań 2008, 499 S. . . . . . . . . . ..............................

MONIKA KOWALONEK-JANCZAREK, Boris Blahak, Clemens Piber (Hg.): Deutsch als fachbezogene Fremdsprache in Grenzregionen. Ekonóm, Bratislava 2008, 322 S. . . . . . . .

LUIZA CIEPIELEWSKA-KACZMAREK, Sambor Grucza (Hg.): W kręgu teorii i praktyki lingwistycznej. Księga jubileuszowa poświęcona Profesorowi Jerzemu Lukszynowi z okazji 70. rocznicy urodzin. Wydawnictwo Uniwersytetu Warszawskiego, Warszawa 2007, 367 S. . . . . . . . .

AGNIESZKA PAWŁOWSKA, Kultury i języki poznawać - uczyć się - nauczać / Kulturen und Sprachen verstehen - lemen - lehren. Pod redakcją / Herausgegeben von Anna Jaroszewska, Marta Torenc. Uniwersytet Warszawski, Instytut Germanistyki, Warsza-

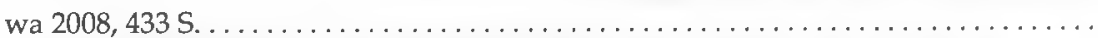

BEATA MikOŁAJCZYK, Konrad Ehlich, Dorothee Heller (Hg.): Die Wissenschaft und ihre Sprachen. 2006. Peter Lang Verlag, Bern / Berlin / Bruselles / Frankfurt a.M. / New York / Oxford / Wien. (= Linguistic Insights. Studies in Language and Communi-

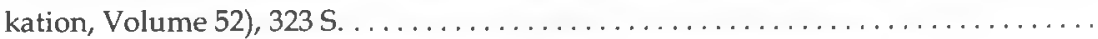




\section{REPORTS}

LUIZA CIEPIELEWSKA-KACZMAREK, Lesen und Leseverstehen in der DaF-Didaktik

MAGDAlena JUREWICZ, PAWEE RYBSZLEGER, Bericht über die Internationale wissenschaftliche Konferenz des Verbandes Polnischer Germanisten (VPG) ..........

AGNIESZKA PAWŁOWSKA, Bericht über die internationale Konferenz Reflexion als Schlüsselphänomen der gegenwärtigen Fremdsprachendidaktik

GRAŻYNA ZENDEROWSKA-KORPUS, Das linguistisch-hochschuldidaktische Kolloquium „Fachsprachenpropädeutik im Germanistikstudium" 



\title{
Die Entwicklung interner grammatischer Regeln im gesteuerten Fremdsprachenunterricht
}

\author{
Developing internal rules \\ in instructed second language acquisition
}

\begin{abstract}
ABSTRACr. Internal rules of grammar exist in the language irrespective of how they are described by the linguists. This paper is an attempt to answer the question in what way internal rules develop both in the mother tongue and in instructed second language acquisition. The author refers to the hierarchy of processing procedures, chunk related and cognitively based research and demonstrates how this development may be supported in instructed second language acquisition.
\end{abstract}

Keywords: rules of grammar, grammar acquisition, second language acquisition.

Der Begriff der Systemhaftigkeit der Sprache ist seit de Saussure der zentrale Grundsatz der Linguistik. Dieser Grundsatz hat unsere Vorstellung von der Grammatik stark geprägt. Da das so gesehene Sprachsystem stark an den Schriftsprachnormen orientiert ist, wird die Grammatik allgemein als das bloße Lernen von Konjugations- und Deklinationsformen und die Satzanalyse im traditionellen Sinne verstanden. So definierte Grammatik wäre eigentlich als bewusst gelernte, mit Regeln (und Ausnahmen) ausgestattete Variante der Sprache zu bezeichnen.

Dabei ist es entscheidend, dass die linguistische Beschreibung der strukturellen Beziehungen im Satz keine eindeutigen Erkenntnisse über die „internen" Regeln, d.h. die Regeln der mentalen Grammatik ${ }^{1}$, darstellt - egal, ob es sich um eine Mutter- oder Fremdsprache handelt.

${ }^{1}$ Als Synonyme zu der mentalen Grammatik werden auch „subjektive Grammatik", „internalisierte Grammatik“ und „Lernergrammatik“ gebraucht (der letzte Begriff in Bezug auf die fremdsprachliche Grammatik). 
Die mentale Grammatik wird von der Psycholinguistik als Teil des mentalen Lexikons gesehen. Das mentale Lexikon besteht aus sprachlichen Einheiten ${ }^{2}$; daher umfasst das sprachliche Wissen zwei Hauptteile: das Wissen sprachlicher Einheiten und das Kombinationswissen. Die Kenntnis der sprachlichen Einheiten nennt man das mentale Lexikon, die Kenntnis der Kombinationsmöglichkeiten die mentale Grammatik (Dietrich 2007: 27).

So wären die internen Regeln - aus psycholinguistischer Sicht gesehen als Prinzipien zu definieren, die festlegen, wie aus elementaren Einheiten komplexere zusammengesetzt sind. Nach Dietrich (2007: 28) umfassen diese Prinzipien die Kombination von:

- Konzepten zu komplexeren semantischen Gebilden, Bedeutungsstrukturen genannt,

- syntaktischen Einheiten zu komplexen syntaktischen Gebilden (Phrasen und Sätze),

- einfachen lexikalischen Einheiten zu komplexeren Einheiten (Wortformen und neue Wörter),

- lautlichen Einheiten zu komplexeren Einheiten (phonologische Phrasen und die intonatorische Einheit der Äußerung).

Die internen grammatischen Regeln existieren in der Sprache unabhängig von ihrer Beschreibung durch die Linguisten. Nach Willis Edmondson (2002: 53) stellen die internen Regeln eine Art kognitive Ausstattung dar, auf deren Grundlage eine Sprache mit bestimmten Normen konform verwendet werden kann.

In Bezug auf die Fremdsprachengrammatik bedeutet die Beherrschung der internen Regeln, dass der Lerner imstande ist, seine expliziten grammatischen Kenntnisse im Sprachhandeln umzusetzen. Aber mit diesen expliziten grammatischen Kenntnissen stehen die internen Regeln selbstverständlich in keinem direkten Zusammenhang.

Warum der moderne Fremdsprachenunterricht vom traditionellen Grammatikunterricht wenig profitieren kann, hat in diesem Kontext Erwin Tschirner zutreffend dargelegt:

Die statischen Regeln der Systemlinguistik, die aus sorgfältig formulierten schriftlichen Texten abgeleitet werden, sind damit qualitativ anderer Art als die dynamischen Regeln der mentalen Grammatik, die durch Verarbeitungsprozesse in sprachlichen Interaktionssituationen erworben werden. Grammatische Kompetenz lässt sich nicht dadurch erwerben, dass man grammatische Regeln lernt, anwendet und durch Üben automatisiert (Tschirner 2001: 111).

2 Für die Bezeichnung der sprachlichen Einheit gebraucht Dietrich den Begriff der lexikaIischen Einheit (Dietrich 2007: 30 u.a.). In der Literaturforschung wird auch der Begriff "Listem" gebraucht; Listeme sind sprachliche Größen, die von einem Individuum in seinem mentalen Lexikon gespeichert werden müssen (Di Sciullo/Williams 1987, Gallman 1990). 
Die Fremdsprachenforscher sind sich darüber einig, dass explizite Grammatikregeln nur eine indirekte Wirkung auf die Entwicklung der grammatischen Kompetenz haben und sich vor allem zur kognitiven Kontrolle der Richtigkeit einer schon vorliegenden Konstruktion eignen.

In dieser Hinsicht sind die Ansätze interessant, die bei der systematischen Behandlung im Unterricht ein sog. induktives Verfahren vorschlagen (Zimmermann 1984). Diese Methode kann man als einen Versuch bezeichnen, den Erwerb interner Regeln zu initiieren und zu gestalten, evtl. auch zu beschleunigen. Diesbezüglich verwendet Erik Kwakernaak den Begriff des gezielten Induktionstrainings; es sei möglich, den Grammatikunterricht so zu gestalten, dass die Lerner nach Regelmäßigkeiten - z.B. in der Pluralbildung von Nomen - suchen, auch aus eigener Initiative (Kwakernaak 1996). Diese aktiv analysierende Haltung den grammatischen Phänomenen gegenüber fördere den Lernprozess weitestgehend. Daraus ergäben sich bestimmte Vorteile für den Lernenden:

Bei der praktischen Arbeit an der Konstruktion von Induktionsübungen (...) habe ich jede einzuführende Grammatikregel sorgfältig analysieren müssen, um entscheiden zu können, welches Element von den Lernern induziert werden kann und welches Element bzw. welche Elemente zur Lenkung 'verschenkt', also vorgegeben werden müssen. Dabei habe ich erfahren, dass Regeln, aus der Lemerperspektive gesehen, oft nicht so einfach sind, wie sie dem Sprachlehrer bzw. Lehrwerkautor vor der Analyse erschienen. Der induktive Ansatz macht dem Lehrwerkautor und möglicherweise auch dem mit dem betreffenden Lehrwerk arbeitenden Lehrer stärker bewusst, was bei der Anwendung einer Grammatikregel dem Lerner an kognitiven Handlungen zugemutet wird (Kwakernaak 1996: 287).

Obwohl Kwakernaak auf die Nachteile der Induktion im Lernprozess hinweist (großer Zeitaufwand, Gefahr, dass der Lerner falsche Schlussfolgerungen zieht), betont er, dass mit induktivem Verfahren längerfristig sehr gute Lernergebnisse erzielt werden können. ${ }^{3}$

Im Grunde genommen lassen sich seine Ausführungen auf die Feststellung zurückführen, dass es beim Sprachenlernen bestimmte Erwerbssequenzen gibt. In der Fremdsprachenforschung gibt es Konzepte, für die dieser Begriff von zentraler Bedeutung ist. Ihnen zufolge läuft der Erwerb einer Fremdsprache in universell festgelegten Sequenzen ab. Die grammatischen Regeln werden in einer festen Reihenfolge erworben, die für jede Sprache eigen ist. Diese Reihenfolge muss auf keinen Fall mit der unterrichtlichen Grammatikprogression übereinstimmen.

${ }^{3}$ Induktives Verfahren weist viele Analogien zu der auf Lernerfragen basierenden Methode (Eckerth 1998) auf, die die sog. Hypothesenbildung und -überprüfung zum Ziel hat. 
Anders formuliert: Es gibt in jeder Sprache eine feste Hierarchie von Sprachlernstufen und keine von ihnen können die Lerner überspringen. Manfred Pienemann spricht in diesem Kontext von einer hierarchy of processing procedures (Pienemann 1998). In seiner Processability Theory, der Levelts (1989) Sprachproduktionsmodell zugrunde liegt, heißt es, dass es sich beim Erwerb der L1-Syntax - im Rahmen der kognitiven Verarbeitungsprozesse - kaum um die Übertragung auf den Zweitsprachenerwerb handeln könne. Die L2 werde nach einer hierarchy of processing procedures erworben, egal auf welche L1 sie aufgebaut wird (u. a. Pienemann 1998, Pienemann \& Håkansson 1999). ${ }^{4}$ Viele empirische Befunde bestätigen diese Theorie (Ellis 1989, Vainikka \& Young-Scholten 1996a u. 1996b, Eubank 1996).

Der Antwort auf die Frage, wie die internen Regeln entwickelt werden, kommt man mit chunks-bezogenen Forschungen näher. Sie haben ergeben - so die zentrale These - dass chunks die Grundlage des Spracherwerbs bilden. Chunks werden zuerst gespeichert und dann zunehmend auf die Regelmäßigkeit analysiert; aufgrund dieser Analyse, die unbewusst ist und automatisch stattfindet, wird schrittweise ein Regelsystem aufgebaut. Praktisch bedeutet dies, dass Grammatik durch Lexik gelernt/erworben wird, was sowohl den Erst- wie auch den Fremdsprachenerwerb betrifft (Sharwood Smith 1993, Ellis 1996, Wray/Perkins 2000, Aguado 2002).

Diese Feststellungen entsprechen dem kognitiv-linguistischen Forschungsansatz vollständig, wonach alle sprachlichen Kategorien, eingeschlossen grammatische Strukturen, konzeptuell und semantisch motiviert sind (z.B. Langacker 1999, Bellavia 2007; Radden / Dirven 2007).

Interessante Erkenntnisse für die Fremdsprachendidaktik liefert ein breit angelegtes internationales Forschungsprojekt "Deutsch in Genfer Schulen". Dieses über zwei Jahre laufende Projekt fokussierte auf Grammatikerwerb in der Fremdsprache Deutsch bei französischsprachigen Schülern. ${ }^{5}$ Anhand der darin gewonnenen Ergebnisse konnte die These bestätigt werden, dass es im Grammatikunterricht so etwas wie Erwerbsschritte gibt. Das Projekt bringt auch detaillierte Erkenntnisse zur Frage der Erwerbssequenzen und belegt, dass sie sich nicht immer mit der grammatischen Progression von Lehrbüchern und Lehrplänen decken.

${ }_{4}$ Pienemanns Processability Theory ist eine weiterentwickelte Form seiner teachability hypothesis (1984).

${ }^{5}$ Der vollständige Titel dieses Projekts lautet "Grammatikerwerb im Fremdsprachenunterricht untersucht am Beispiel Deutsch als Fremdsprache an Genfer Schulen"; die genaue Beschreibung des Forschungsprojekts ist nachzulesen bei Diehl/Christen/Leunenberger/Pelvat/ Studer 2000. 
Was die Erwerbsschritte im Grammatikunterricht betrifft, haben die Forscherinnen und Forscher drei Phasen unterschieden (nach Diehl 2002):

I. In der ersten Phase werden aus dem Input einzelne Elemente ausgefiltert, memorisiert und als chunks verwendet ${ }^{6}$ (Guten Morgen, ins Kino, Es war einmal..., Wie geht es dir?). Kennzeichnend ist aber, dass chunks auch den Beginn der Auseinandersetzung mit jeder neuen Form oder Struktur charakterisieren. Die Formen "heute gehe ich", "morgen kaufe ich" werden schon korrekt gebraucht, obwohl die Subjekt-Verb-Inversion noch lange nicht beherrscht wird.

II. In der zweiten Phase verhalten sich die Schüler so, als wollten sie alle möglichen Formen ausprobieren, was zu Formulierungen wie "ich mage", "ich habe gehelft" führt. Offensichtlich wird diese Phase durch eine Überlastung des Gedächtnisspeichers ausgelöst: Es sind schon so viele chunks memorisiert, dass der Schüler nach "ökonomischeren" Speicherungsmethoden sucht, d.h. nach Gesetzen, die es ihm ermöglichen, sich besser in diesem "Chaos" zu orientieren.

Wie die Autorinnen betonen, ist diese Phase für den gesteuerten Grammatikerwerb am aufschlussreichsten.

III. Erst in der letzten Phase sind die Schüler imstande, neue FormFunktion-Beziehungen wahrzunehmen und das "Morphem- und Strukturinventar" auszudifferenzieren, sodass es schrittweise zur graduellen Verbesserung der sprachlichen Kompetenzen der Lerner kommt.

Wie bereits erwähnt, wurde aufgrund der im Projekt gesammelten Daten eine detaillierte Darstellung der Erwerbssequenzen in drei unterschiedlichen Grammatikbereichen vorbereitet. Zu betonen ist, dass die Schwierigkeitshierarchie, die sich daraus ergibt, stellenweise von der unterrichtlichen Progression „abweicht".

In der folgenden Tabelle wird die Erwerbsreihenfolge im Bereich der Verbalmorphologie, der Satzmodelle und des Kasussystems dargestellt (nach Diehl et al. 2000: 364):

Sequenzen im Grammatikerwerb: Zusammenfassung

\begin{tabular}{|c|c|c|}
\hline A Verbalbereich & B Satzmodelle & C Kasus (ohne Präposition) \\
\hline $\begin{array}{l}\text { I } \\
\text { Präkonjugale Phase } \\
\text { (Infinitive; } \\
\text { Personalformen nur als chunks) }\end{array}$ & $\begin{array}{l}\text { I } \\
\text { Hauptsatz } \\
\text { (Subjekt-Verb) }\end{array}$ & $\begin{array}{l}I \\
\text { Ein-Kasus-System } \\
\text { (nur N-Formen) }\end{array}$ \\
\hline
\end{tabular}

${ }^{6}$ Der Begriff chunk wurde von George A. Miller eingeführt und für Größenordnungen verwendet, die als Ganzes verarbeitet werden (Miller 1956). 


\begin{tabular}{|c|c|c|}
\hline A Verbalbereich & B Satzmodelle & C Kasus (ohne Präposition) \\
\hline $\begin{array}{l}\text { II } \\
\text { Konjugation der regelmäßi- } \\
\text { gen Verben im Präsens }\end{array}$ & $\begin{array}{l}\text { II } \\
\text { Koordinierte Haupt- } \\
\text { sätze } \\
\text { W-Fragen } \\
\text { Ja/Nein-Fragen }\end{array}$ & \\
\hline $\begin{array}{l}\text { III } \\
\text { Konjugation der unregelmä- }\end{array}$ & \multirow{2}{*}{$\begin{array}{l}\text { III } \\
\text { Distanzstellung } \\
\text { (Verbalklammer) }\end{array}$} & \\
\hline $\begin{array}{l}\text { Bigen Verben im Präsens } \\
\text { Modalverb + Infinitiv }\end{array}$ & & $\begin{array}{l}\text { II } \\
\text { Ein-Kasus-System }\end{array}$ \\
\hline \multirow{2}{*}{$\begin{array}{l}\text { IV } \\
\text { Auxiliar + Partizip }\end{array}$} & \multirow{2}{*}{$\begin{array}{l}\text { IV } \\
\text { Nebensatz }\end{array}$} & \\
\hline & & \multirow[b]{2}{*}{$\begin{array}{l}\text { III } \\
\text { Zwei-Kasus-System } \\
\text { Nominativ + Objektkasus } \\
\text { (N-Formen + beliebig ver- } \\
\text { teilte A- und D-Formen }\end{array}$} \\
\hline $\begin{array}{l}\text { V } \\
\text { Präteritum }\end{array}$ & $\begin{array}{l}\mathrm{V} \\
\text { Inversion } \\
\text { (X-Verb-Subjekt) }\end{array}$ & \\
\hline $\begin{array}{l}\text { VI } \\
\text { übrige Formen }\end{array}$ & $\begin{array}{l}\text { Erwerb der Satzmodelle } \\
\text { I-V } \\
\text { abgeschlossen }\end{array}$ & $\begin{array}{l}\text { IV } \\
\text { Drei-Kasus-System } \\
\text { Nominativ + Akkusativ }+ \\
\text { Dativ } \\
\text { (N-Formen }+ \text { A-Formen }+D- \\
\text { Formen) }\end{array}$ \\
\hline
\end{tabular}

Aus der Tabelle ist ersichtlich, dass das Erwerbstempo in den drei Bereichen unterschiedlich ist. Die Phasen der Verbalflexion werden relativ schnell vollzogen und am schwierigsten ist der Erwerb des Kasussystems:

(...) Über einen langen Zeitraum hinweg, während im Verbalbereich und bei den Satzmodellen der Erwerb in großen Schritten vorangeht, stagniert der Kasuserwerb. Die Genfer Schüler begnügen sich mit dem Nominativ; Dativ und Akkusativ erscheinen fast ausschließlich in Präpositionalphrasen als vermutlich weitgehend memorisierte Temporal- oder Lokalangaben. Als nächster Schritt folgt ein ziellos wirkendes Experimentieren mit dem gesamten Kasusmorpheminventar ohne eine erkennbare funktionale Differenzierung. Diese "turbulente" Phase endet mit einer Klärung von Nominativ als dem Subjektskasus einerseits und einer Sammelkategorie "oblique Kasus" andererseits, d.h. Akkusativ und Dativ werden als freie Varianten behandelt. Damit ist zunächst ein Zwei-Kasus-System erreicht, das für die Mehrzahl der Genfer Maturanden das maximal Erreichbare in ihrer zehnjährigen schulischen Deutschkarriere darstellt (Diehl 2002: 150). 
Den Grund dafür sieht die am Projekt beteiligte Erika Diehl einerseits in der Komplexität der Kasusmorphologie und ihrer funktionalen Zuweisung, andererseits in der geringen kommunikativen Relevanz der Kasusmarkierung (Diehl 2002: 152).

Höchst interessant sind auch die Erkenntnisse, die dabei hinsichtlich der potentiellen Analogie zum muttersprachlichen Deutscherwerb zutage treten. Von einer solchen Analogie ist in Bezug auf den Erwerb im Verbalbereich wie auch im Bereich des Kasussystems die Rede, da sich dieselben Erwerbsstrategien und Erwerbsabfolgen wie beim L1- und natürlichen L2-Erwerb beobachten lassen. Beim Satzmodellerwerb ist dies nicht der Fall: Während die deutschen L1-Sprecher sich die Verb-Zweit-Stellung sehr früh aneignen, deutet der Erwerbsverlauf bei den französischen Schülern scheinbar auf einen Transfer aus der Erstsprache hin und könnte als allmähliche Ablösung vom Basismodell (Subjekt-Verb) interpretiert werden.

Diehl stellt die These auf, dass die Übertragung aus der L1 nur teilweise, d.h. nicht in allen Bereichen stattfindet, nämlich nur in solchen, in denen die Lerner von dieser Übertragung intuitiv einen Erfolg erwarten. Fest steht, dass dies auf den Bereich der Lexik anwendbar ist, in der Grammatik auch auf den Bereich der Satzstruktur, nicht aber auf die Morphologie.

Auf die Frage, ob die in der Tabelle dargestellten Erwerbssequenzen auch für Deutschlerner mit anderen Muttersprachen als Französisch gelten, wäre so zu antworten:

Für den Erwerb von Verbal- und Nominalmorphologie könnten dieselben Sequenzen dargestellt gelten, eine Annahme, die auch durch den Erwerbsverlauf in der L1 gestützt wird. Die Sequenz für den Satzmodellerwerb hingegen muss vermutlich für jedes Sprachenpaar neu ermittelt werden, da hierfür offensichtlich die jeweilige Beschaffenheit der Basisstrukturen von L1 und L2 determinierend ist. Beide Vorgehensweisen wären auf kognitive Mechanismen zurückzuführen, die den menschlichen Spracherwerb - wenn nicht menschlichen Wissenserwerb generell - regieren: einmal die progressive Erschließung komplexer Systeme durch Vereinfachung/Generalisierung und Ausdifferenzierung, zum anderen der Transfer bereits integrierter Wissensbestände auf neue Sachverhalte (Diehl 2002: 155).

Mit den oben dargestellten Ausführungen wird das angesprochene Problem nur allgemein skizziert. Da der Wortschatz einer Sprache kognitiv motiviert ist und die Grammatik als Korrelation von Sprachformen und Bedeutungen gesehen werden kann, lassen die kognitiv fundierten Forschungsansätze einen beträchtlichen Erkenntnisfortschritt erwarten. Nun sollen lediglich einige offene Fragen gestellt werden, die evtl. den Anstoß zu weiteren Studien geben könnten: 
- In welchem Verhältnis steht die im induktiven Verfahren gewonnene grammatische Regel zu der "entsprechenden“ internen Regel? Lässt sich das im induktiven Verfahren gewonnene grammatische Wissen als interne Regel bezeichnen?

- Wie viele chunks (evtl. Sequenzen von nichtformelhaftem Charakter) muss der Lerner "ansammeln“, um eine Regel - in Bezug auf jedes grammatische Phänomen - ableiten zu können?

- Woran lässt sich erkennen, dass ein Lerner schon eine konkrete interne Regel entwickelt hat?

- Gibt es besser und schlechter entwickelte interne Regeln? Welches Maß soll eigentlich angewendet werden?

- Können interne Regeln vergessen werden?

\section{LITERATURVERZEICHNIS}

Aguado, K., 2002. Formelhafte Sequenzen und ihre Funktionen für den L2-Erwerb. In: Zeitschrift für Angewandte Linguistik 37, S. 27-50.

Bellavia, E., 2007. Erfahrung, Imagination und Sprache. Die Bedeutung der Metaphern der Alltagssprache für das Fremdsprachenlernen am Beispiel der deutschen Präpositionen. Tübingen: Narr.

Diehl E./Christen H./Leunenberger S./Pelvat I./Studer T., 2000. Grammatikunterricht - Alles für der Katz? Untersuchungen zum Zweitsprachenerwerb Deutsch. Tübingen: Niemeyer.

DiehI E., 2002. Grammatikerwerb im Fremdsprachenunterricht - ein Widerspruch in sich?. In: Börner W./Vogel K. (Hrsg.), Grammatik und Fremdsprachenerwerb. Kognitive, psycholinguistische und erwerbstheoretische Perspektiven. Tübingen: Narr, 143-156.

Dietrich, R., 2007, Psycholinguistik. Stuttgart: Metzler.

Di Sciullo A.M./Williams E., 1987. On the Definition of Word. Cambridge, MA: MIT Press.

Doughty C./Williams J., 1998. Focus on form in classroom second language acquisition. Cambridge: Cambridge University Press.

Eckerth J., 1998. Kognitive Aspekte sprachbezogener Lernerfragen. Interaktion und Kognition im Deutsch-als-Fremdsprache-Unterricht. Baltmannsweiler: Schneider.

Edmondson, W.J., 2002. Wissen, Können, Lernen - kognitive Verarbeitung und Grammatikentwicklung. In: Börner W./Vogel K. (Hrsg.), Grammatik und Fremdsprachenerwerb. Kognitive, psycholinguistische und erwerbstheoretische Perspektiven. Tübingen: Narr, 51-70.

Ellis, N., 1996. Sequencing in SLA: Phonological memory, chunking and points of order. In: Studies In Second Language Acquisition 18, 91-126.

Ellis, R., 1989. Are classroom and naturalistic acquisition the same? A study of the classroom acquisition of German word order rules. In: Studies in Second Language Acquisition 11, 305-25.

Eubank, L., 1996. Negation in early German-English interlanguage: more 'valueless features' in the L2 initial state. In: Second Language Research 10, 84-93.

Gallman, P., 1990. Wortschreibung und Schemakonstanz. In: Zeitschrift für Germanistik 5/90, 513-523.

Kwakernaak, E., 1996. Grammatik im Fremdsprachenunterricht. Geschichte und Innovationsmöglichkeiten am Beispiel Deutsch als Fremdsprache in den Niederlanden. Amsterdam, Atlanta: Rodopi. 
Langacker, R.W., 1999. Grammar and Conceptualization. Berlin, New York: Mouton de Gruyter. Miller, George A., 1956. The Magical Number Seven, Plus or Minus Two. In: Psychological Review $63,81-87$.

Levelt, W.J.M., 1989. Speaking: From intention to articulation. Cambridge, MA: MIT Press.

Pienemann, M., 1984. Psychological constraints on the teachability of languages. In: Studies in Second Language Acquisition 6, 186-214.

Pienemann, M., 1998. Language processing and second language development: Processability Theory. Amsterdam, Philadelphia: John Benjamins.

Portmann-Tselikas, P. R., 2003. Aufmerksamkeit statt Automatisierung. Zur Rolle des Wissens im Grammatikunterricht. In: gf-journal 2/2003.

Radden, G./Dirven, R., 2007. Cognitive English Grammar. Cognitive Linguistics in Practice. Amsterdam, Philadelphia: John Benjamins.

Sharwood Smith, M., 1993. Input enhancement in instructed SLA: Theoretical bases. In: Studies In Second Language Acqusition 15, 165-179.

Tschirner, E., 2001. Kompetenz, Wissen, mentale Prozesse: Zur Rolle der Grammatik im Fremdsprachenunterricht. In: Funk, H./Koenig, M. (Hrsg.), Kommunikative Didaktik in Deutsch als Fremdsprache - Bestandsaufnahme und Ausblick. Festschrift für Gerhard Neuner, München: iudicium, 106-25.

Vainikka, A./Young-Scholten, M., 1996a. Gradual development of L2 phrase structure. In: Second Language Research 12, 7-39.

Vainikka, A./Young-Scholten, M., 1996b. The early stages in adult L2 syntax: additional evidence Romance speakers. In: Second Language Research 12, 140-76.

Wray, A./Perkins, M.R., 2000. The functions of formulaic language: An integrated model. In: Language \& Communication, 1-28.

Zimmermann, G., 1984. Erkundungen zur Praxis des Grammatikunterrichts. Frankfurt am Main: Diesterweg. 
